\title{
The Role of Behind Folds Visualizing Techniques and Technologies in Improving Adenoma Detection Rate
}

\author{
K.E. van Keulen, MD
}

\section{E. Soons, $M D$}

P.D. Siersema, prof, $d r^{*}$

\author{
Address \\ "Department of Gastroenterology and Hepatology, Radboud University Medical \\ Center, Nijmegen, The Netherlands \\ Email: Peter.Siersema@radboudumc.nl \\ Published online: 22 July 2019 \\ (C) The Author(s) 2019
}

\section{Electronic supplementary material}

K.E. van Keulen and E. Soons are co-first authors.

This article is part of the Topical Collection on Endoscopy

The online version of this article (https://doi.org/10.1007/s11938-019-00242-5) contains supplementary material, which is available to authorized users.

Keywords Colorectal cancer $\cdot$ Adenoma detection rate $\cdot$ Adenoma miss rate $\cdot$ Behind folds visualizing technique . Cap-assisted colonoscopy · EndoRings · Endocuff Vision • Extra Wide Angle View (EWAVE) · Full Spectrum Endoscopy (FUSE) - Third Eye Panoramic cap · Third Eye Retroscope · Scope retroflexion · Systematic review · Meta-analysis

\begin{abstract}
Purpose of review Colorectal cancer is one of the most common malignancies in the Western world and is thought to develop from premalignant polyps. Over the past decade, several behind folds visualizing techniques (BFTs) have become available to improve polyp detection. This systematic review and meta-analysis aims to compare BFTs with conventional colonoscopy (CC).

Recent findings In the past five years, 14 randomized controlled trials (RCTs) including 8384 patients comparing different BFTs with CC were published. The overall relative risks for adenoma detection rate, polyp detection rate, and adenoma miss rate comparing BFTs with $\mathrm{CC}$ were 1.04 (95\% confidence interval [CI] 0.98-1.10; $P=0.15), 1.03(95 \%$ CI 0.98-1.09; $P=0.28)$, and 0.70 (95\% CI $0.46-1.05 ; P=0.08$ ), respectively. Other quality metrics for colonoscopy were not significantly different between BFT-assisted colonoscopy and $\mathrm{CC}$ either.

Summany This meta-analysis of RCTs published in the past five years does not show a significant benefit of BFTs on any of the important quality metrics of colonoscopy. The lack of additional effect of BFTs might be due to improved awareness of colonoscopy quality metrics and colonoscopy skills among endoscopists combined with improvements of conventional colonoscope technology.
\end{abstract}




\section{Introduction}

Colorectal cancer (CRC) is the third most commonly diagnosed malignancy and the fourth leading cause of death in the Western world [1, 2]. CRC usually develops from focal changes within benign, precancerous polyps. The two most well-known types of polyps with malignant potential include conventional adenomas and sessile serrated adenomas/polyps (SSA/SSPs) [3, 4]. It is estimated that $60 \%$ of all CRCs arise from adenomas and an additional 35\% from SSA/SSPs [5]. Colonoscopy aims to early detect and remove these precancerous polyps. Although colonoscopy is generally considered to be the most accurate screening modality, a substantial number of polyps are still being missed. A meta-analysis of tandem colonoscopy studies reported a pooled miss rate for polyps of any size of $22 \%$ and an adenoma miss rate (AMR) of $26 \%$ for diminutive polyps $(1-5 \mathrm{~mm}$ ) [6]. Missed lesions have the opportunity to develop into cancer. It is thought that at least $50 \%$ of all interval carcinomas (iCRCs; defined as a cancer diagnosed between screening and post-screening surveillance colonoscopies) arise from missed lesions during colonoscopy [7].

Currently, the most commonly used quality marker for conventional colonoscopy (CC) is adenoma detection rate (ADR), which has been shown to inversely correlate with the incidence of CRC. A landmark study by Kaminski et al. reported a higher risk of iCRC if the ADR with CC was less than 20\% [8]. In addition, Corley et al. concluded that each $1.0 \%$ increase in ADR was associated with a $3.0 \%$ decreased risk of iCRCs and a $5.0 \%$ decreased risk of a fatal iCRC [ $9 \bullet \bullet]$.

Several factors may have a negative effect on ADR, including shorter withdrawal time, poor bowel preparation, and the inability to visualize the proximal side of haustral folds, flexures, and rectal- and ileocecal valves [10]. Incomplete inspection has been quantified in a study that estimated that $13.4 \%$ of the colonic surface remains unseen using a standard forward-viewing colonoscope, despite adequate bowel preparation [11].

Over the past 5-10 years, several techniques and technologies have been developed that aim to better detect premalignant colorectal lesions. One of the methods to improve ADR is the use of imageenhanced endoscopy, including flexible spectral imaging color enhancement (FICE), i-Scan, narrow-band imaging, and chromoendoscopy. A recent meta-analysis concluded that none of these image-enhanced endoscopy technologies was able to confidently claim that they improved ADR when compared with standard/highdefinition white light endoscopy [12]. Another method to improve ADR is to use one of the so called behind folds visualizing techniques and technologies (BFTs), including a cap, the Endocuff Vision, the EndoRings, and Third Eye Panoramic cap, all four mounted on the tip of the scope. Other BFTs are integrated into the endoscope, such as the Full Spectrum Endoscopy (FUSE) colonoscope, the G-Eye colonoscope, and the more recently introduced Extra Wide Angle View (EWAVE) colonoscope. Finally, retroflexion within the colon can be performed with the Third Eye Retroscope and some conventional colonoscopes with $180^{\circ}$ bending capacity. These BFTs have in common that they aim to improve polyp and adenoma detection by increasing visualization and inspection of the mucosal surface.

Below, these techniques and technologies will be discussed in more detail:

\section{Cap-assisted colonoscopy}

For cap-assisted colonoscopy, a transparent cap is attached onto the distal tip of the colonoscope. This cap is able to depress the colonic folds to optimize visualization of lesions that are located behind mucosal folds. Furthermore, it allows keeping some distance between the colonoscope and the colonic mucosa which prevents a so called red-out sing and unintentionally suctioning of normal mucosa, mimicking the presence of a polyp.

\section{Endocuff Vision}

The Endocuff Vision (Arc Medical Design Ltd., Leeds, England) is the second generation of the original Endocuff. It is a disposable device that can be attached onto the distal end of a colonoscope. Unlike the firstgeneration Endocuff, the Endocuff Vision has only a single row of soft, flexible fingerlike projections. The projections are hinged at their base to prevent interference with the forward movement during insertion. When withdrawing the colonoscope, the "fingers" flare out and flatten the mucosal folds.

\section{EndoRings}

The EndoRings (Endo-Aid, Caesarea, Israel) is a silicone-rubber device that also fits onto the distal end of a colonoscope. It originally consisted of three circular rows of wings that flare out when withdrawing the colonoscope and flatten the colonic folds. The most recent version of the EndoRings consists of only two rows of wings. 
Full Spectrum Endoscopy

The FUSE system (Endo-Choice, Alpharetta, GA) maximizes the field of view to $330^{\circ}$ by adding two lateral viewing lenses to the single forward-viewing lens. Each camera projects to a different screen, resulting in a total of three screens. The FUSE colonoscope is no longer available, since the company has been acquired by another company that decided not to further commercialize it.

G-Eye

The G-Eye (Smart Medical Systems Ltd., Ra'anana, Israel) is composed of a cylindrical balloon that is permanently integrated into the distal portion of the colonoscope shaft. The balloon is in the deflated state during colonoscope insertion and can be inflated upon withdrawal resulting in flattening of the mucosal folds, centralization of the endoscopic view and reduction of scope slippage when performing polypectomy.

Extra Wide Angle View

The most recent prototype of the EWAVE colonoscope (Olympus Medical Systems, Tokyo, Japan) provides an increased field of view to $235^{\circ}$ and consists of a standard $147^{\circ}$ forward-viewing lens with two additional $42.5^{\circ}$ lateral-backward viewing lenses. Views from all lenses are simultaneously displayed on a video monitor as a single endoscopic image. A death angle of three degrees between the lenses is filtered out in the composited image. Third Eye Retroscope/Panoramic cap
The Third Eye Retroscope (Avantis Medical Systems Inc., Sunnyvale, CA) was used to be a disposable imaging device that was inserted through the working channel of the colonoscope. When placed approximately $40 \mathrm{~mm}$ in front of the tip, it bents to $180^{\circ}$ providing a retrograde view on a second screen. The Third Eye Retroscope is no longer commercially available, as its use in daily clinical practice was considered to be impractical. Its successor, the Third Eye ${ }^{\circledast}$ Panoramic $^{\mathrm{TM}}$ device (TEP, Avantis Medical Systems) is a plastic cap containing two side-viewing cameras that can be attached to the tip of any standard colonoscope. This results in three distinct images that are projected on a single screen, expanding the viewing angle to $>300^{\circ}$.

Scope retroflexion

The term retroflexion refers to making a U-turn with the distal bendable portion of the colonoscope. By doing this, the lens is looking backward, and the insertion tube is visible, but more importantly, the proximal aspects of the mucosal folds can be inspected, providing an increased field of visualization.

All the abovementioned BFTs aim to improve the field of inspection compared with CC. In the following, we performed a systematic review with the objective to assess the additional value of the abovementioned BFTs compared with CC.

\section{Materials and methods}

\section{Protocol and registration}

This study was registered in the Prospero database of systematic reviews (CRD42019117433) on November 21, 2018.

\section{Eligibility criteria}

We only included randomized controlled trials (RCTs) that compared the efficacy of BFTs with CC in adult patients referred for screening, surveillance, or diagnostic colonoscopy. Studies that included patients with inflammatory bowel disease or familial colorectal cancer syndromes were excluded. Studies were also excluded if they were published before January first, 2013, or if a previous version of a BFT was used. In addition, animal studies (conference), abstracts, clinical trial registries, studies not reporting original data, and studies that did not report ADR and/or adenoma miss rate (AMR) were excluded. For practical reasons, we only included studies published in the English language. 
We performed a systematic electronic search using three bibliographic databases: PubMed, Embase (Ovid interface), and the Cochrane Library on November 23, 2018. Terms used for the data search included "polyp," "adenoma," "colonoscopy," "endoscopy," "balloon assisted," "cap assisted," "Endocuff," "EndoRing," "extra wide angle view (EWAVE)," "Full spectrum colonoscopy (FUSE)," "G-Eye," "retroflex," and "Third Eye." These search terms were composed in collaboration with an experienced medical librarian. Snowballing was used to identify additional articles. The full search strategy for this study can be found in Supplementary Table 1.

\section{Study selection}

EndNote X8 (Clarivate Analytics, Philadelphia, PA, USA) was used for deduplication after which all remaining articles were exported to the browserbased systematic review management program Rayyan QCRI (Qatar Computing Research Institute, Hamad Bin Khalifa University, Qatar) [13]. The selected articles and abstracts were independently evaluated by two researchers (KK and ES) to determine eligibility of each study. The full text of all eligible studies was reviewed by both authors. Disagreements were resolved by discussion between the two researchers. When disagreement remained, this was ultimately resolved through discussion with a third reviewer (PS).

The primary outcome was ADR. Secondary outcomes were AMR, polyp detection rate (PDR), advanced adenoma detection rate (AADR), cecal intubation rate (CIR), cecal intubation time (CIT), withdrawal time (WT), and bowel preparation.

ADR, PDR, and AADR were defined as the percentage of patients in who at least one adenoma, polyp, or advanced adenoma was detected. AMR and AAMR were defined as the ratio between the total numbers of adenomas or advanced adenomas, respectively, detected during the second colonoscopy procedure over the total number of adenomas detected in both colonoscopy procedures. In tandem studies, ADR and PDR were calculated using only the data of the first colonoscopy procedure. CIR was defined as the proportion of patients in which the cecum was reached in all patients evaluated in each group. Withdrawal time was defined as the time required for inspection of the mucosa (not including time for diagnostic or therapeutic interventions).

\section{Data extraction and statistical analysis}

Eligible studies were divided into two categories; (1) RCTs in which patients were allocated to either BFT-assisted colonoscopy or CC to compare ADR and (2) RCT-tandem colonoscopy studies, in which two same-day colonoscopies were performed in each patient starting with either BFT-assisted colonoscopy or $\mathrm{CC}$ and then followed by the other technique, to compare AMR.

All data were extracted and double checked for errors by both researchers. For all included studies, relative risks (RR) were calculated for ADR, PDR, and AMR with 95\% confidence intervals (CI) and $P$ values. Pooled estimates were 
calculated per device and per category (BFT versus CC) to establish overall rates. RevMan version 5.3.5. (The Nordic Cochrane Centre, Copenhagen, Denmark) was used to calculate relative risks and generate pooled estimates and 95\% confidence intervals (CI) using a random effects model for meta-analysis and prevalence. Cecal intubation time and withdrawal time were compared with a weighted independent $t$ test. Time outcomes were calculated with IBM SPSS 22 (IBM. Corp., Armonk, NY, USA).

Heterogeneity for pooled estimates was assessed using $I^{2}$ statistics. Low, moderate, and high heterogeneity was defined as $I^{2}$ values of $\geq 25 \%, 50 \%$, or $75 \%$, respectively. All $I^{2}$ values were calculated with RevMan. Funnel plots were inspected visually for asymmetry of the main outcome.

Risk of bias assessment

Risk of bias for all RCTs was assessed using the Cochrane Collaboration Risk of Bias tool [14]. To make the tool more specific for our review, we did not include the domains "blinding of investigators" and "blinding of outcome(s)" in our final risk assessment, since blinding is obviously not possible with BFT-assisted colonoscopy. Two investigators (KK and ES) independently assessed the remaining relevant study domains. Disagreements were resolved through discussion. The risk of bias was graded as low, high, or unclear.

\section{Results}

\section{Systematic search}

The systematic search identified 2475 citations. Ultimately, 14 RCTs were included in the final analysis which evaluated five different BFTs: cap-assisted colonoscopy (CAC) ( $n=3$ studies), Endocuff Vision ( $n=2$ studies), EndoRings ( $n=2$ studies), FUSE ( $n=6$ studies), and G-Eye ( $n=2$ studies) (Fig. 1 ). Rex et al. compared Endocuff, EndoRings, and FUSE with CC in one RCT [15••]. Evaluation of Endocuff was excluded in our analysis since the previous version of the Endocuff Vision was used. Over the past five years, no RCTs were published in which the scope retroflexion, the EWAVE, and the Third Eye Retroscope/ Panoramic cap were compared with CC.

\section{Study characteristics}

The 14 RCTs included comprised a total of 8384 patients. Studies included in our analysis originated from the USA, the UK, Italy, Germany, Israel, Spain, Denmark, Japan, and The Netherlands. ADR was the primary outcome in nine

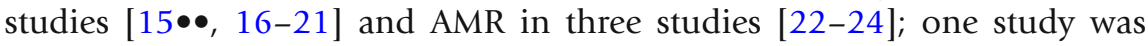
powered for both ADR and AMR [25]. The remaining studies had another primary outcome. Patients underwent colonoscopy for screening (64.2\%), surveillance $(21.1 \%)$, or a diagnostic indication (14.6\%) (Table 1$)$.

\section{Primary outcome}

Although all 14 studies reported ADR, only eight studies were adequately powered to detect a significant difference in ADR between BFT-assisted colonoscopy and CC (Fig. 2 and Supplementary Table 2). Three studies, including 1761 colonoscopy patients, compared CAC with CC yielding a pooled RR of 1.01 (95\% CI 0.90-1.14; $P=0.57 ; I^{2}=0 \%$ ) $[16,26,27]$. Two studies, including 


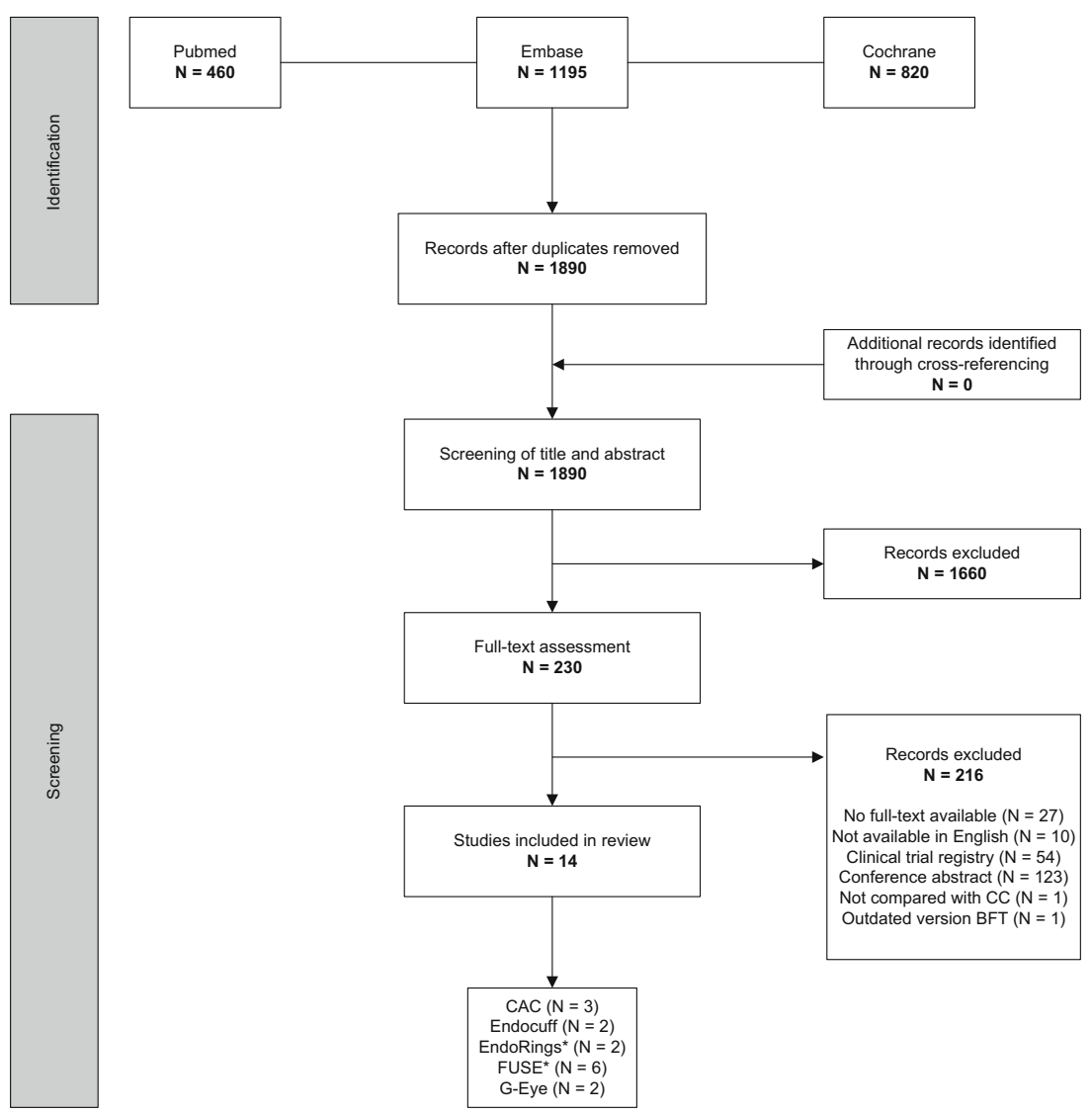

Fig. 1. PRISMA diagram. Flowchart representing literature search and elements of systematic review (identification and screening). CC conventional colonoscopy, BFT behind folds visualizing techniques, CAC cap-assisted colonoscopy, FUSE Full Spectrum Endoscopy. * Rex et al. (2018) compared EndoRings and FUSE with CC in one study.

2303 patients, compared Endocuff Vision-assisted colonoscopy with CC which yielded a pooled RR of 1.05 (95\% CI $\left.0.90-1.22 ; P=0.56 ; I^{2}=66 \%\right)[17,28]$. EndoRings-assisted colonoscopy was compared with CC in two studies, including 1517 patients, yielding a pooled RR of 0.98 (95\% CI 0.89-1.07; $P=0.62$; $\left.I^{2}=0 \%\right)[15 \bullet \bullet, 18]$. Six studies, including 2209 patients, compared FUSE with CC resulting in a pooled RR of 1.04 (95\% CI $\left.0.94-1.15 ; P=0.49 ; I^{2}=40 \%\right)$ $[15 \bullet \bullet, 18-20,22,23]$. Two studies, including 909 patients, evaluated G-Eye colonoscopy yielding a significant RR of $1.30\left(95 \%\right.$ CI $1.11-1.51, P<0.05 ; I^{2}=$ $0 \%$ ) in favor of G-Eye.

The overall RR comparing all BFTs with CC resulted in a pooled RR of 1.04 (95\% CI $0.98-1.10 ; P=0.15 ; I^{2}=38 \%$ ). No difference in ADR was found between studies powered for ADR and those with another primary outcome.

\section{Secondary outcomes}

\section{Polyp detection rate and advanced adenoma detection rate}

Polyp detection rate was reported in nine studies $(n=6003)$. Pooled analysis resulted in a RR of $1.03\left(95 \%\right.$ CI $\left.0.98-1.09 ; P=0.28 ; I^{2}=48 \%\right)$ (Supplementary 


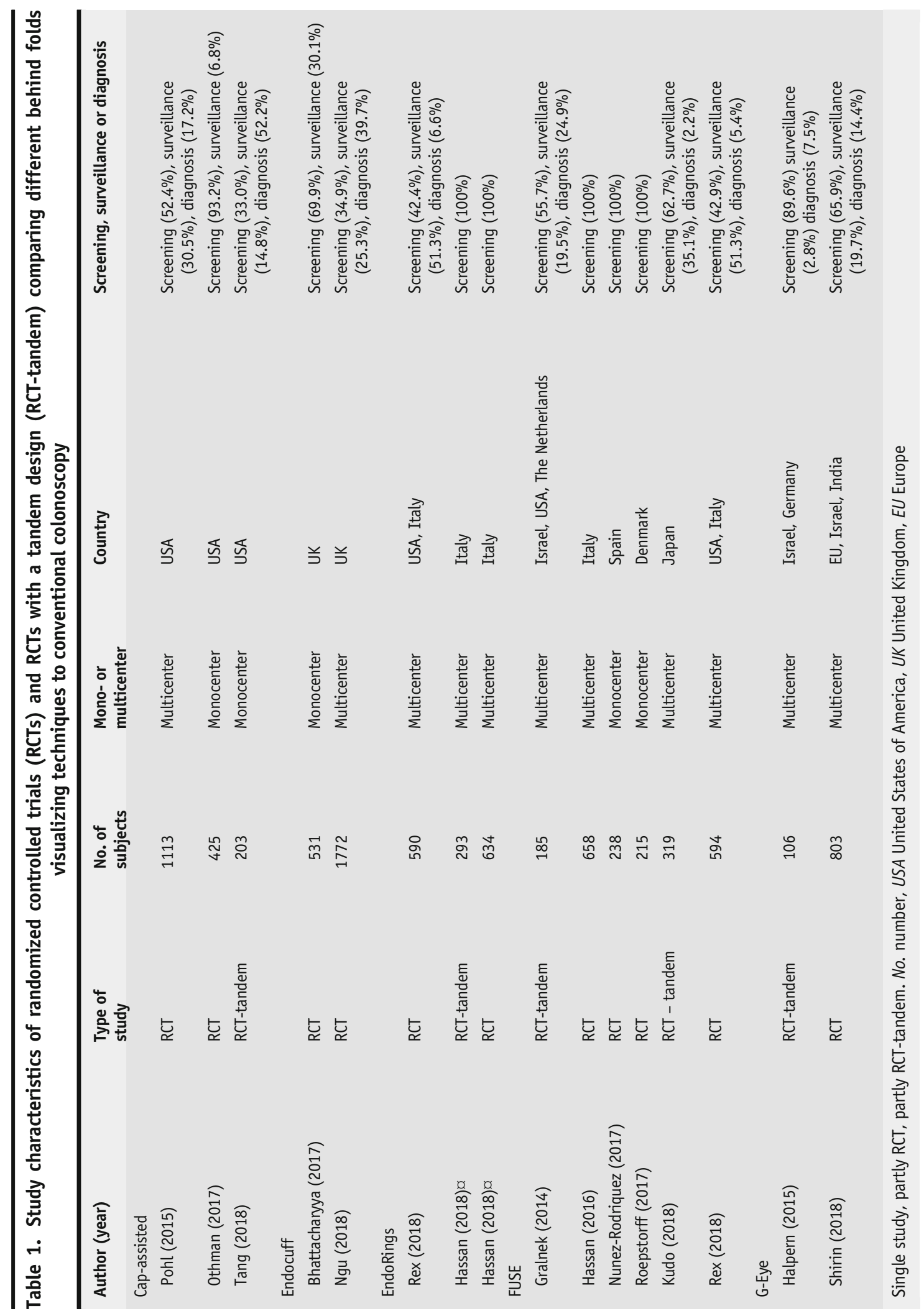




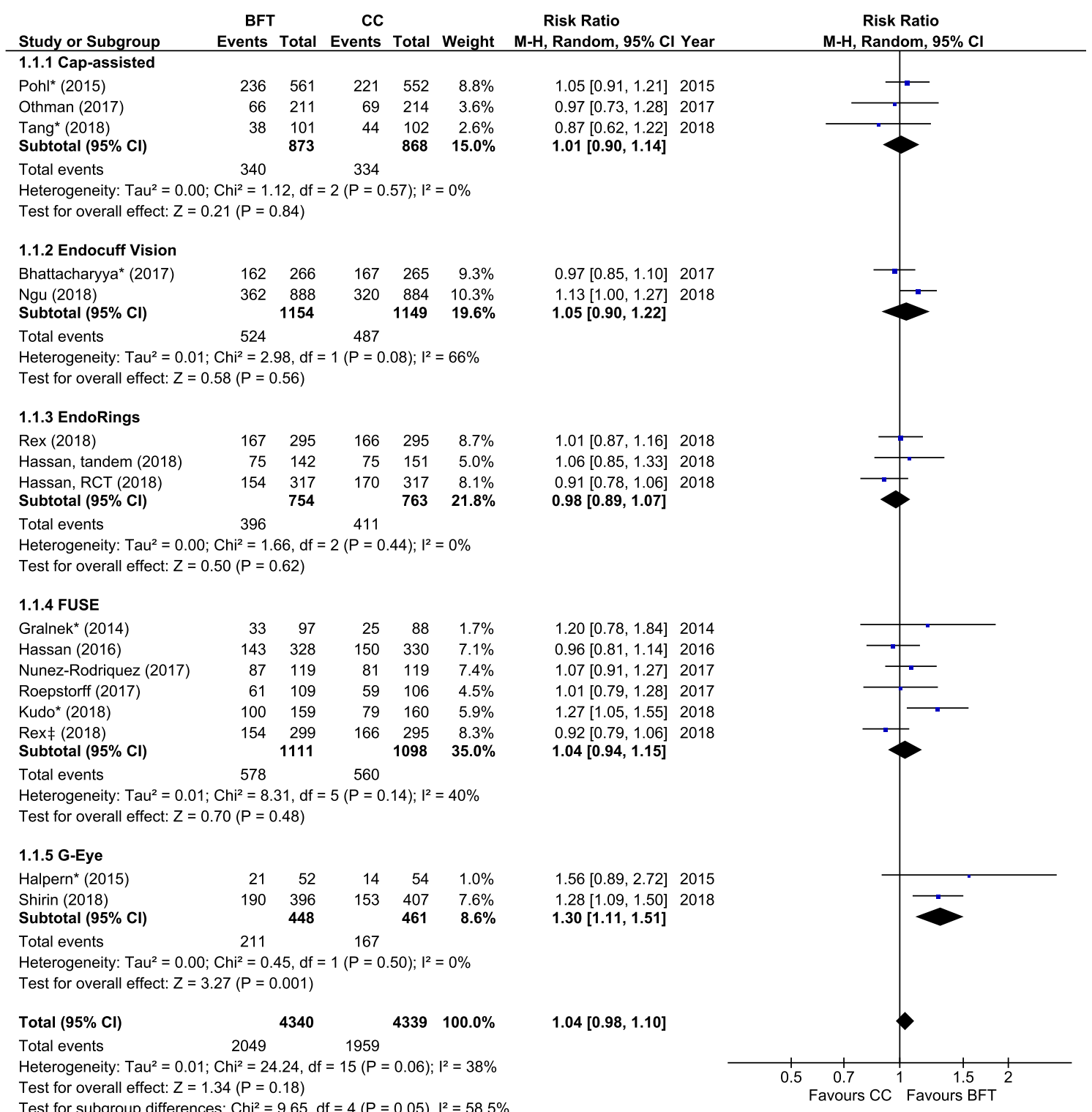

Fig. 2. Forest plot for adenoma detection rate (ADR) with behind folds visualizing techniques (BFTs) versus conventional colonoscopy (CC). Total studies: 13; 8384 subjects. CI confidence interval, FUSE Full Spectrum Endoscopy. *Study not powered for detecting a difference in ADR. ${ }^{\ddagger} 0$ ne study investigating multiple BFTs.

Fig. 1) $[15 \bullet \bullet, 16,17,19-21,25,26,28]$. Six studies reported results on AADR $(n=5144)$. Only Othman et al. reported a significant benefit of CAC (RR 2.13, $95 \%$ CI 1.03-4.41) [16]. Pooled analysis of all studies combined resulted in a RR of 0.98 (95\% CI $\left.0.83-1.14 ; P=0.75 ; I^{2}=26 \%\right)$.

\section{Adenoma miss rate and advanced adenoma miss rate}

Four studies performed tandem back-to-back colonoscopy procedures evaluating EndoRings, FUSE, and G-Eye [22-25]. In terms of AMR, only Halpern et al. 
showed a significant advantage of G-Eye colonoscopy over CC (RR 0.17, 95\% CI 0.05-0.53, $P<0.05)$ [24]. The overall pooled RR for AMR for use of BFTs was 0.70 (95\% CI 0.46-1.05; $P=0.08 ; I^{2}=67 \%$ ) (Supplementary Fig. 2). AAMR was reported in three studies, but none reported a significant difference (Supplementary Table 3). Of all missed lesions, 5.9\% (6/102) and 5.3\% (10/187) were advanced adenomas in the BFT and CC groups, respectively.

\section{Cecal intubation rate and time}

CIR was below the recommended $90 \%$ in two studies [20, 26]. Tang et al. [26] investigated if CAC could improve CIR among gastroenterology fellows and reported a significant improvement of CIR and CIT for CAC compared with CC. In contrast, Roepstorff et al. [20] reported a significant difference in CIR and CIT in favor of CC compared with FUSE colonoscopy. Mean pooled CIT was significantly shorter in the BFT, 7.0 and $7.2 \mathrm{~min}$, respectively $(P<0.05$, mean difference $0.21 \mathrm{~min}$ ).

\section{Withdrawal time}

WT was significantly different in four studies; in three studies, WT was longer in the BFT group [21, 24, 27], and in 1 study, WT was longer in the CC group [28]. Mean WT was 7.8 versus 7.7 min for BFT and CC, respectively $(P<0.05$, mean difference $0.08 \mathrm{~min}$ ).

\section{Bowel preparation}

Different bowel preparation scales were used among studies; no study reported a significant difference between the intervention and control arm.

\section{Risk of bias}

Risk of bias evaluation for individual studies is shown in Supplementary Table 4. Four studies were graded as low risk, seven studies as moderate risk, and three as high risk of bias. The funnel plot for ADR did not suggest that publication bias was present (Supplementary Fig. 3).

\section{Heterogeneity}

Heterogeneity was moderate for the pooled RR of the Endocuff and the overall pooled RR of AMR (due to divergent results in Halpern et al. [24]) and for the test for subgroup differences. Heterogeneity for all remaining outcomes was low.

\section{Discussion}

This systematic review assessed the additional value of various BFTs compared with CC in 8384 individual patients reported in 14 RCTs. The overall conclusion was that ADR did not improve significantly when using one of the BFTs compared with CC (RR 1.04, 95\% CI 0.98-1.10, $P=0.18$ ). Additionally, our results did not show a significant difference in overall AMR either. 
Due to the small number of tandem colonoscopy studies, no definite conclusions can be drawn but the results suggested that the use of BFTs could well result in a reduced risk of missing non-advanced adenomas, although the difference was not statistically significant. The clinical relevance of detecting more non-advanced lesions remains unknown. Of the factors known to influence ADR, only the weighted WT differed significantly in favor of BFTs. However, the mean difference was only $0.08 \mathrm{~min}$ which seems unlikely to be clinically relevant.

Our results show no overall effect of BFTs on ADR, the principal colonoscopy quality indicator $[29,30]$. This finding is in line with a recent meta-analysis by Facciorusso et al. [31] who compared the effect of CAC, Endocuff (old version), and EndoRings with CC. This meta-analysis also included RCTs published before 2013 and those with previous versions of add-one devices. The only device found to significantly improve ADR in this analysis was the Endocuff. In contrast to our results, Castaneda et al. [32] reported a 35\% increase in ADR comparing both optical (e.g., FUSE and Third Eye colonoscopy) and mechanical BFTs (e.g., Endocuff, EndoRings, and G-Eye colonoscopy) with CC. This increase in ADR may well be explained by the fact that half of the studies included in this analysis evaluated the Endocuff (previous version). The use of the Endocuff showed a $40 \%$ increase in ADR. Moreover, a major part of the data from this meta-analysis was derived from conference abstracts (not peer-reviewed) and observational and cohort studies. This may well explain the high heterogeneity $\left(I^{2}=62 \%\right)$ that was reported in this review. We excluded conference abstracts and observational and cohort studies from our review, which likely explains the rather low heterogeneity in our review.

In terms of AMR, our analysis did not show an additional effect of BFTs compared with CC. This finding clearly conflicts with the results of two previous meta-analyses that reported a $19-35 \%$ reduction in overall AMR with BFTs $[32,33]$. However, both meta-analyses included studies that were excluded in our analysis either because they were published before $2013[34,35]$, written in German [36•], and used a previous version of the Endocuff or EndoRings [37-39], or because they did not use CC as a comparator [40]. Similarly to the results on ADR, the Endocuff results were the most positive in these analyses.

This systematic review has several strengths. A major advantage of the review is that it was performed according to the PRISMA guideline, which, among other things, included a prepublished protocol, an up-to-date literature search and two authors independently screening all references and assessing risk of bias of the included studies. As we excluded nonrandomized studies, we were able to reduce the heterogeneity between different studies. At least some of the remaining heterogeneity can likely be explained by the variance between the different BFTs, indicating that the type of BFT may well modify the ADR effect size and that not all BFTs are equally effective.

There are also some limitations, related to both our review and the individual studies. First, we decided to exclude a significant number of studies because older versions of the Endocuff and EndoRings were used, full-text peer-reviewed papers were not available, or studies were published 
in the non-English literature, such as studies in the German [41-44], Italian [45], Japanese [46], Korean [47-49], and Spanish [50] languages. Having excluded these studies may have led to selection bias and a lower generalizability of our results. Second, only a limited number of RCTs were available that compared the various BFTs head-to-head with CC. This was particularly true for tandem studies, which resulted in a limited number of studies reporting on AMR and AAMR, and therefore, the clinical importance of the reported outcomes remains unclear. Third, all included studies were unblinded RCTs which could have resulted in performance and detection bias. Nonetheless, due to the nature of the intervention (colonoscopy), blinding of endoscopists is obviously not easily implemented. It is indisputable that the ideal tandem study is done with two endoscopists, in which the endoscopists are blinded for each other's results. Finally, one of the main pitfalls of these types of studies is the Hawthorne effect, stimulating participating endoscopists to do their utmost best to find adenomas, particularly when using the technique or technology under investigation resulting in an overestimated ADR in the experimental study arms.

It is of interest that most pilot studies of ADR-improving devices show significant improvements in outcomes such as ADR and AMR [36•, 51-54].. However, these "positive" results can often not be replicated during follow-up in well-designed RCTs that are frequently performed in a multicenter design, instead of the single-center design of the initial pilot studies. This discrepancy could also be due to the Hawthorne effect and to the study design. In this regard, it is important to keep in mind that conventional colonoscopes constantly improve in quality and performance. Moreover, the increased awareness of important colonoscopy quality metrics (e.g., bowel preparation, CIR, and WT) and colonoscopists' skills are also known to improve ADR $[8,9 \bullet \bullet]$.

Although the results of this meta-analysis may look disappointing, it is important to keep in mind that most of these studies were performed by highly experienced endoscopists, while it may well be that BFTs are indeed beneficial for a subgroup of less experienced colonoscopists and/or those with baseline low ADRs. This hypothesis should be assessed in future studies.

In conclusion, this systematic review shows that the use of BFTs does not have a clear effect on improving overall ADR and AMR. Nevertheless, BFTs may well improve the detection of non-advanced polyps and lesions, although the clinical importance of this finding is currently unknown and needs to be further studied. Finally, if an endoscopist decides to use one of the currently available BFTs in daily clinical practice, particularly the G-Eye and Endocuff Vision seem currently the most interesting technologies.

\section{Compliance with Ethical Standards}

\section{Conflict of Interest}

K.E. van Keulen and E. Soons declare no conflict of interest.

P.D. Siersema currently receives research support from and is in the Advisory Board of Pentax Medical - Japan. 


\section{Human and Animal Rights and Informed Consent}

All reported studies/experiments with human or animal subjects performed by the authors have been previously published and complied with all applicable ethical standards (including the Helsinki Declaration and its amendments, institutional/national research committee standards, and international/national/institutional guidelines).

Open Access This article is distributed under the terms of the Creative Commons Attribution 4.0 International License (http://creativecommons.org/licenses/by/4.0/), which permits unrestricted use, distribution, and reproduction in any medium, provided you give appropriate credit to the original author(s) and the source, provide a link to the Creative Commons license, and indicate if changes were made.

\section{References and Recommended Reading}

Papers of particular interest, published recently, have been highlighted as:

- $\quad$ Of importance

$\bullet \quad$ Of major importance

1. Arnold M, Sierra MS, Laversanne M, et al. Global patterns and trends in colorectal cancer incidence and mortality. Gut. 2017;66:683-91.

2. Endoscopy ASfG. Colorectal Cancer Statistics. Volume 2018. https://www.asge.org/home/about-asge/ newsroom/media-backgrounders-detail/colorectalcancer-statistics, 2014.

3. Vogelstein B, Fearon ER, Hamilton SR, et al. Genetic alterations during colorectal-tumor development. N Engl J Med. 1988;319:525-32.

4. Leggett B, Whitehall V. Role of the serrated pathway in colorectal cancer pathogenesis. Gastroenterology. 2010;138:2088-100.

5. Snover DC. Update on the serrated pathway to colorectal carcinoma. Hum Pathol. 2011;42:1-10.

6. van Rijn JC, Reitsma JB, Stoker J, et al. Polyp miss rate determined by tandem colonoscopy: a systematic review. Am J Gastroenterol. 2006;101:343-50.

7. Pohl H, Robertson DJ. Colorectal cancers detected after colonoscopy frequently result from missed lesions. Clin Gastroenterol Hepatol. 2010;8:858-64.

8. Kaminski MF, Regula J, Kraszewska E, et al. Quality indicators for colonoscopy and the risk of interval cancer. N Engl J Med. 2010;362:1795-803.

9.• Corley DA, Jensen CD, Marks AR, et al. Adenoma detection rate and risk of colorectal cancer and death. $\mathrm{N}$ Engl J Med. 2014;370:1298-306

This study is one of the landmark studies that evaluated the relationship between adenoma detection rate and colonoscopy outcomes.

10. Rees CJ, Rajasekhar PT, Rutter MD, et al. Quality in colonoscopy: European perspectives and practice. Expert Rev Gastroenterol Hepatol. 2014;8:29-47.

11. East JE, Saunders BP, Burling D, et al. Surface visualization at CT colonography simulated colonoscopy: effect of varying field of view and retrograde view. Am J Gastroenterol. 2007;102:2529-35.

12. Omata F, Ohde S, Deshpande GA, et al. Image-enhanced, chromo, and cap-assisted colonoscopy for improving adenoma/neoplasia detection rate: a systematic review and meta-analysis. Scand J

Gastroenterol. 2014;49:222-37.

13. Ouzzani M, Hammady H, Fedorowicz Z, et al. Rayyana web and mobile app for systematic reviews. Syst Rev. 2016;5:210.

14. Higgins JP, Altman DG, Gotzsche PC, et al. The Cochrane Collaboration's tool for assessing risk of bias in randomised trials. Bmj. 2011;343:d5928.

$15 . \bullet$ Rex DK, Repici A, Gross SA, et al. High-definition colonoscopy versus Endocuff versus EndoRings versus full-spectrum endoscopy for adenoma detection at colonoscopy: a multicenter randomized trial. Gastrointest Endosc. 2018;88:335-344.e2

This study compared different BFTs within the same study.

16. Othman MO, Zhang D, Elhanafi S, et al. Cap-assisted colonoscopy increases detection of advanced adenomas and polyps. Am J Med Sci. 2017;353:367-73.

17. Ngu WS, Bevan R, Tsiamoulos ZP, et al. Improved adenoma detection with Endocuff Vision: the ADENOMA randomised controlled trial. Gut. 2018.

18. Hassan C, Senore C, Radaelli F, et al. Full-spectrum (FUSE) versus standard forward-viewing colonoscopy in an organised colorectal cancer screening programme. Gut. 66:1949-55.

19. Nunez-Rodriguez H, Diez-Redondo P, Perez-Miranda $\mathrm{M}$, et al. Role of Full-spectrum Endoscopy in colorectal cancer screening: randomized trial. J Clin Gastroenterol. 2017.

20. Roepstorff S, Hadi SA, Rasmussen M. Full spectrum endoscopy (FUSE) versus standard forward-viewing endoscope (SFV) in a high-risk population. Scand J Gastroenterol. 2017;52:1298-303.

21. Shirin H, Shpak B, Epshtein J, et al. G-EYE colonoscopy is superior to standard colonoscopy for increasing adenoma detection rate: an international randomized controlled trial (with videos). Gastrointest Endosc. 2018. 
22. Gralnek IM, Siersema PD, Halpern Z, et al. Standard forward-viewing colonoscopy versus full-spectrum endoscopy: an international, multicentre, randomised, tandem colonoscopy trial. Lancet Oncol.

2014; 15:353-60.

23. Kudo T, Saito Y, Ikematsu H, et al. New-generation fullspectrum endoscopy versus standard forward-viewing colonoscopy: a multicenter, randomized, tandem colonoscopy trial (J-FUSE Study). Gastrointest Endosc. 2018.

24. Halpern Z, Gross SA, Gralnek IM, et al. Comparison of adenoma detection and miss rates between a novel balloon colonoscope and standard colonoscopy: a randomized tandem study. Endoscopy. 2015;47:238-44.

25. Hassan C, Senore C, Manes G, et al. Diagnostic yield and miss rate of EndoRings in an organized colorectal cancer screening program: the SMART (Study Methodology for ADR-Related Technology) Trial. Gastrointest Endosc. 2018.

26. Tang Z, Zhang DS, Thrift AP, et al. Impact of capassisted colonoscopy on the learning curve and quality in colonoscopy: a randomized controlled trial. Gastrointest Endosc. 2018;87:723-732.e3.

27. Pohl H, Bensen SP, Toor A, et al. Cap-assisted colonoscopy and detection of Adenomatous Polyps (CAP) study: a randomized trial. Endoscopy. 2015;47:891-7.

28. Bhattacharyya R, Chedgy F, Kandiah K, et al. Endocuffassisted vs. standard colonoscopy in the fecal occult blood test-based UK Bowel Cancer Screening Programme (E-cap study): a randomized trial. Endoscopy. 2017;49:1043-50.

29. Rex DK, Schoenfeld PS, Cohen J, et al. Quality indicators for colonoscopy. Gastrointest Endosc.

2015;81:31-53.

30. Kaminski MF, Thomas-Gibson S, Bugajski M, et al. Performance measures for lower gastrointestinal endoscopy: a European Society of Gastrointestinal Endoscopy (ESGE) quality improvement initiative. United European Gastroenterol J. 2017;5:309-34.

31. Facciorusso A, Del Prete V, Buccino RV, et al. Comparative efficacy of colonoscope distal attachment devices in increasing rates of adenoma detection: a network meta-analysis. Clin Gastroenterol Hepatol.

2018;16:1209-1219.e9.

32. Castaneda D, Popov VB, Verheyen E, et al. New technologies improve adenoma detection rate, adenoma miss rate, and polyp detection rate: a systematic review and meta-analysis. Gastrointest Endosc. 2018;88:209222.e11.

33. Gkolfakis P, Tziatzios G, Facciorusso A, et al. Metaanalysis indicates that add-on devices and new endoscopes reduce colonoscopy adenoma miss rate. Eur J Gastroenterol Hepatol. 2018.

34. Hewett DG, Rex DK. Cap-fitted colonoscopy: a randomized, tandem colonoscopy study of adenoma miss rates. Gastrointest Endosc. 2010;72:775-81.

35. Leufkens AM, DeMarco DC, Rastogi A, et al. Effect of a retrograde-viewing device on adenoma detection rate during colonoscopy: the TERRACE study. Gastrointest Endosc. 2011;73:480-9.
36. Rey JW, Dumcke S, Haschemi J, et al. G-EYE advanced colonoscopy for improved polyp detection rates - a randomized tandem pilot study with different endoscopists. Z Gastroenterol 2018.

This tandem colonoscopy study charged endoscopists after each procedure to compensate for observer-related bias. Observer-related bias is a big problem in unblinded endoscopy studies which is why we feel that the ideal tandem study is done with two different endoscopists.

37. Dik VK, Gralnek I, Segol O, et al. Comparing standard colonoscopy with EndoRingsTM colonoscopy: a randomized, multicenter tandem colonoscopy studyinterim results of the clever study. United European Gastroenterol J. 2014;1:A489.

38. Triantafyllou K, Polymeros D, Apostolopoulos P, et al. Endocuff-assisted colonoscopy is associated with a lower adenoma miss rate: a multicenter randomized tandem study. Endoscopy. 2017;49:1051-60.

39. De Palma GD, Giglio MC, Bruzzese D, et al. Cap cuffassisted colonoscopy versus standard colonoscopy for adenoma detection: a randomized back-to-back study. Gastrointest Endosc. 2018;87:232-40.

40. Papanikolaou IS, Apostolopoulos P, Tziatzios G, et al. Lower adenoma miss rate with FUSE vs. conventional colonoscopy with proximal retroflexion: a randomized back-to-back trial. Endoscopy. 2017;49:468-75.

41. Escher M. Cap assisted colonoscopy improves detection of serrated polyps. [German]. Dtsch Med Wochenschr. 2015;140:634.

42. Frieling T, Neuhaus F, Kuhlbusch-Zicklam R, et al. Prospective and randomized study to evaluate the clinical impact of cap assisted colonoscopy (CAC). Z Gastroenterol. 2013;51:1383-8.

43. Herrmann IF, Gadebusch Bondio M, Domagk D, et al. New possibilities with retroflexed functional endoscopy. Hno. 2018;66:527-33.

44. Schmidt-Tanzer W, Eickhoff A. What influences the quality of prevention colonoscopy? Viszeralmedizin. 2014;30:26-31.

45. Manes G, De Nucci G. FUSE - colonoscopy with full spectrum technology. Giornale Italiano di Endoscopia Digestiva. 2015;38:49-50.

46. Takezawa T, Yano T, Sunada K, et al. The technique of colonoscopy by using balloon-assisted endoscopy. [Japanese]. Gastroenterol Endosc. 2014;56:2000-10.

47. Kim HU, Boo SJ, Na SY, et al. Additional polyp detection rate using colonoscopic retroflexion in right colon. Korean J Gastroenterol. 2015;65:90-8.

48. Shin SJ. New colonoscopy devices to improve colon neoplasm detection. Korean J Gastroenterol. 2016;68:293-6.

49. Choi SW, Park HS, Lee JS, et al. Efficacy of hood-cap assisted colonoscopy; comparison with conventional colonoscopy. Intest Res. 2012;10:280-8.

50. Peniche Moguel PA, Zarate Guzman AM, Garcia Guerrero $\mathrm{V}$, et al. Comparison of the prevalence of adenomas detected by CAP-assisted colonoscopy versus standard colonoscopy. Endoscopia. 2016;28:143-7. 
51. Tsiamoulos Z, Wilson A, Saunders B. Endocuff assisted colonoscopy significantly improve adr comparing to cap assisted colonoscopy: a randomised study (DETECT). Gut. 67:A38.

52. DeMarco DC, Odstrcil E, Lara LF, et al. Impact of experience with a retrograde-viewing device on adenoma detection rates and withdrawal times during colonoscopy: the Third Eye Retroscope study group.

Gastrointest Endosc. 2010;71:542-50.

53. Dik VK, Gralnek IM, Segol O, et al. Multicenter, randomized, tandem evaluation of EndoRings colonoscopy-results of the CLEVER study. Endoscopy. 2015;47:1151-8.
54. Bronzwaer M, Dekker E, Weingart VP, et al. Feasibility, safety and accuracy of the extra wide angle view (EWAVE) colonoscope for the detection of colorectal lesions. Gastrointest Endosc. 85:AB503.

\section{Publisher's Note}

Springer Nature remains neutral with regard to jurisdictional claims in published maps and institutional affiliations. 